\title{
USO DA PROGRAMAÇÃO LINEAR PARA ESTIMAR O PADRÃO DE CULTURA DO PERÍMETRO IRRIGADO NILO COELHO ${ }^{1}$
}

\author{
José Dantas Neto², Carlos Alberto Vicira de Azevedo² e José Antônio Frizzone ${ }^{3}$
}

\begin{abstract}
RESUMO
Os administradores de perímetros irrigados devem planejar com antecedência como será utilizada a água disponível, dada a sua acentuada demanda. Sabe-se que existe uma interrelação entre a demanda e a disponibilidade de água, porque os padrões de cultivo tendem a se ajustar à disponibilidade. Esta pesquisa teve como objetivo estudar o padrão de cultivo do Perímetro Irrigado Senador Nilo Coelho, PE, utilizando um modelo de programação linear. A técnica de programação linear tem sido amplamente usada para representar esse tipo de sistema de produção, principalmente com fins de planejamento em áreas novas ou para melhorar projetos de irrigação já em operação. O modelo estudado é uma formulação-padrão de programação linear, cuja função-objetivo consistiu em maximizar a receita líquida do projeto, utilizando-se as culturas mais cultivadas nessa área, sob regime de irrigação. As restrições à função-objetivo foram volume de água mensal, volume de água anual, terra e mercado. A maximização dos lucros da área de colonização do projeto Nilo Coelho, obtida com o modelo de programação linear, foi de US\$ 22.634.044, para o seguinte padrão de cultivo: 保ão Phaseolus (714 ha), melancia (714 ha), pimentão (714 ha), tomate (4281 ha), cebola (357 ha) e banana ( 818 ha). A disponibilidade anual apresentou um custo de oportunidade igual a 0,28 (US $\left.\$ . \mathrm{m}^{3}\right)$ e foi a restrição atuante, ao contrário das disponibilidades mensais.
\end{abstract}

Palavras-chave: programação linear, perímetro irrigado, padrão de cultura

\section{USE OF LINEAR PROGRAMMING FOR ESTIMATING CROP PATTERN FOR THE NILO COELHO IRRIGATION DISTRICT}

\begin{abstract}
The irrigation district managers must plan early how available water should be used, given its large demand. It is known that a relationship between demand and water availability exists, because the crop patterns have the tendency to adjust to the water availability. The purpose of this research was to study the crop pattern for the Nilo Coelho, PE irrigation district, using a linear programming model. The linear programming technique has been widely used for representing this kind of production system, mainly for planning purposes in new areas or for improving the irrigation projects already in operation. The studied model is a traditional linear programming formulation, in which the objective function consisted into maximizing the net income of the project using the most cultivated crops in the area, under irrigation conditions. The restrictions to the objective function were the monthly and annual water volume, land and marketing. The maximization of profits in the colonization area of the Nilo Coelho project, given by the linear programming model, was equal to US $\$ 22,634.044$, for the following crop pattern: beans (714 ha); watermelon ( $714 \mathrm{ha}$ ); green pepper ( $714 \mathrm{ha}$ ); tomato (428 ha); onion (357 ha); and banana ( 818 ha). The annual water availability presented an opportunity cost equal to 0.28 (US $\$ . \mathrm{m}^{-3}$ ) and was the strongest restriction, contrary to the monthly availabilities.
\end{abstract}

Key words: linear programming, irrigation district, crop pattern

\footnotetext{
1 Parte da tese do primeiro autor-UINESP, Botucatu, SP, Brasil.

2 Prof. Adjunto - DEAG/CCT / UFPb, Campina Grande, PB, Brasil.

${ }^{3}$ Prol. Assistente Dr. - DER/ESALQ/USP, Piracicaba, SP, Brasil.
} 


\section{INTRODUÇÃO}

Em áreas irrigadas, onde várias culturas em diferentes regimes de irrigação estão competindo por uma quantidade limitada de água, a programação linear (PL) é um excelente instrumento para a alocação ótima desses recursos.

Muitos trabalhos foram desenvolvidos no sentido de selecionar culturas a serem plantadas em determinada área, em condições limitantes de água e técnicas de programação linear têm sido usadas para a resolução de lais problemas. Windsor e Chow (1971) usaram um modelo de PL para selecionarem o tipo de cultura, a área de terra a ser alocada a cada uma, o sistema e a intensidade de irrigação para a produção.

O uso de promoção linear exige o conhecimento de todos os fatores que possam influenciar o funcionamento do sistema a ser otimizado. No caso de projetos de irrigação, diz Albuquerque Filho (1986) que os seguintes fatores estão entre os que mais comumente afetam a operação e os custos dos sistemas, influindo diretamente nos seus benelícios: a) Disponibilidade de recursos naturais (solo/água) adequados; b) Disponibilidade de capital; c) Políticas operacionais adequadas (quando e quanto irrigar); d) Uso de insumo (fertilizantes e defensivos); e) Disponibilidade de mão-de-obra; f) Disponibilidade de equipamentos apropriados; g) Comercialização da produção (armazenamento, preços etc.); h) Energia e custo compatível; i) Restrições legais (concessões) e outros.

Para Baltra (1982) os modelos de programação linear nos projetos de irrigação podem ser utilizados nos seguintes tipos de planejamento agronômico: (I) Otimização de planos de cultura, para otimização dos recursos, podendo-se utilizar, como função econômica, a) Maximização do lucro; b) Otimização do uso da mão-de-obra; c) Otimização do uso da água e d) Otimização do uso das máquinas agrícolas. O modelo usa as restrições: a) Volume de água disponível; b) Demanda de água pelas culturas; c) Custos de produção; d) Terra disponível; e) Receita líquida; e I) Custos de mercado. II) Lâmina e intervalos de irrigação; III) Previsões meteorológicas; IV) Potencial de bacias de captação e balanços hidrológicos.

A Secretaria de Recursos Hidráulicos do México propôs um modelo de programação linear para o planejamento da operação do distrito de irrigação de Santo Domingo, Baixa Califórnia; este modelo apresentou um plano ólimo de culturas, que aumentava a receita líquida e reduzia o volume de extração do aquíifero de abastecimento. Evia (1975) comenta que a partir de 1976, 83\% dos distritos de irrigação do México adotaram modelos similares.

O objetivo geral deste trabalho foi desenvolver um sistema de equações matemálicas, baseado na função de resposta das culturas à água, adaptando-o a um modelo de programação linear; e com este modelo quantificaram-se as variáveis ou as atividades do processo de produção que otimizam a demanda de água e o padrão de cultivo do Projelo de Irrigação Senador Nilo Coelho, Petrolina, PE, de forma a maximizar o lucro da área irrigada pelos colonos.

\section{MATERIAL E MÉTODOS}

O estudo aqui desenvolvido refere-se ao planejamento da irrigação do Projeto Senador Nilo Coelho (PSNC) localizado em Petrolina, PE, e realizado por Dantas Neto (1994). O modelo geral é uma adaptação proposta por Frizzone (1995) cujo objetivo é determinar um padrão ótimo de cultivo, de forma a maximizar o lucro do projeto, decorrente de várias culturas (Tabela 1).

Tabela 1. Épocas de plantio, necessidades de irrigação, produtividade, preço dos produtos e custos de produção para as culturas utilizadas

\begin{tabular}{|c|c|c|c|c|c|c|}
\hline $\mathrm{i}, \mathbf{k}^{*}$ & Cultura & $\begin{array}{l}\text { Época de } \\
\text { plantio }\end{array}$ & $\begin{array}{l}\text { Inrigação } \\
\text { (mm) }\end{array}$ & $\begin{array}{l}\text { Produt. } \\
\left(\mathrm{kg} \cdot \mathrm{ha}^{-1}\right)\end{array}$ & $\begin{array}{l}\text { Preço do } \\
\text { produto } \\
\left(\mathrm{US} \$ \mathrm{~kg}^{-1}\right)\end{array}$ & $\begin{array}{l}\text { Custo de produção } \\
\text { Exceto água } \\
\text { (US\$.ha }{ }^{-1} \text { ) }\end{array}$ \\
\hline 1,1 & F.Phaseolus & maio & 545 & 2669 & 0,58 & 450,0 \\
\hline 1,2 & F. Phascolus & setembro & 545 & 2669 & 0,58 & 450,0 \\
\hline 2,1 & Feijão Vigna & janeiro & 500 & 1253 & 0,54 & 400,0 \\
\hline 3,1 & Melancia & fevereiro & 887 & 33670 & 0,08 & 750,0 \\
\hline 3,2 & Melancia & outubro & 887 & 33670 & 0,08 & 750,0 \\
\hline 4,1 & Milho & maio & 775 & 7555 & 0,15 & 432,0 \\
\hline 4,2 & Milho & dezembro & 775 & 7555 & 0,15 & 432,0 \\
\hline 5,1 & Melāo & fevereiro & 940 & 13679 & 0,16 & 908,0 \\
\hline 6,1 & Pimentão & setembro & 763 & 12226 & 0,15 & 889,0 \\
\hline 7,1 & Tomate & maio & 568 & 54196 & 0,08 & 1646,0 \\
\hline 8,1 & Cebola & fevereiro & 824 & 40227 & 0,20 & 1530,0 \\
\hline 9,1 & Banana & $2^{\circ}$ ano & 2900 & 54278 & 0,18 & 1200,0 \\
\hline & água - U &, $016 \cdot \mathrm{m}^{-3}$ & & & & \\
\hline
\end{tabular}

$*_{i}$ - número inteiro representando a cultura $(i=1,2, \ldots, n)$

$\mathrm{k}$ - número inleiro representando a época de plantio $(\mathrm{k}=1, \ldots, \mathrm{p})$

\section{O modelo geral}

A funçâo objetivo

$\max \mathrm{Z}=\sum_{I=1}^{n} \sum_{K=1}^{P} P_{\mathrm{I}} \mathrm{Y}_{\mathrm{I}} \mathrm{X}_{\mathrm{LK}}-\sum_{I=1}^{n} \sum_{K=1}^{P} C_{\mathrm{I}} \mathrm{X}_{\mathrm{IK}}-\sum_{I=1}^{n} \sum_{K=1}^{P} C_{\mathrm{I}} \mathrm{W}_{\mathrm{I}} \mathrm{X}_{\mathrm{IK}}$

Lin que: $Z$ - renda líquida decorrente do cultivo de n culturas, em USS; $P_{i}$ - preço unitário do produto, em USS.kg ${ }^{1} ; \mathrm{W}_{\mathrm{i}^{-}}$lâmina total de irrigação necessária à cultura $\mathrm{i}$, em num; $\mathrm{C}_{\mathrm{i}}$ - custos de produção da cultura $\mathrm{i}$, nấo incluindo os custos da água, cm US\$.ha ${ }^{1} ; \mathrm{X}_{\mathrm{ik}}$ - árca cultivada $\mathrm{com}$ a i-ćsima cultura, no k-ésimo período, em ha; e $\mathrm{Y}_{\mathrm{i}}$ - produtividade da i-ésima cultura, em kg.ha ${ }^{-1}$.

$$
\text { Restriçốes a que está sujcita a maximização da renda líquida: }
$$

(1) disponibilidade mensal de terra:

$$
\sum_{l=1}^{n} \sum_{K=1}^{P} \mathrm{X}_{\mathrm{IK}} \leq \mathrm{S}_{\mathrm{J}} \quad(\mathrm{J}=1,2, \ldots, 12) ;
$$

(2) balanço de área cultivada em cada mês:

$\sum_{l=1}^{n} \sum_{K=1}^{P} \mathrm{X}_{\mathrm{IK}}=\mathrm{XM}_{\mathrm{J}} \quad(\mathrm{J}=1,2, \ldots, 12)$

(3) balamço de área tolal cultivada de cada cultura:

$\sum_{K=1}^{P} \mathrm{X}_{\mathrm{IK}}=\mathrm{XT}_{1} \quad(\mathrm{I}=1,2, \ldots, 9)$

(4) disponibilidade mensal de água:

$\sum_{l=1}^{n} \sum_{K=1}^{P} W_{\mathrm{IK}} \mathrm{X}_{\mathrm{IK}} \leq \mathrm{V}_{\lrcorner} \quad(\mathrm{J}=1,2, \ldots, 12) ;$

(5) disponibilidade anual de água:

$\sum_{l=1}^{n} \sum_{K=1}^{l} \mathrm{~W}_{\mathrm{IK}} \mathrm{X}_{\mathrm{IK}} \leq \mathrm{VT}$ 
(6) mercado e capacidade de processamento industrial:

$\sum_{K=1}^{P} \mathrm{X}_{\mathrm{IK}} \leq \geq \mathrm{R}_{\mathrm{IK}}(\mathrm{I}=1,2, \ldots 9)$;

(7) não negatividade: $X_{\mathrm{IK}} \geq 0$.

em que: $\mathrm{S}$ - área máxima disponível para cultivo no mês $\mathrm{j}$, em ha; $\mathrm{XM}_{\mathrm{j}}$ - área cultivada no mês $\mathrm{j}$, em ha; $\mathrm{Xl}_{\mathrm{i}}$ - área total cultivada com a cultura $\mathrm{i}$; em ha; $\mathrm{W}_{\mathrm{ik}}{ }^{-}$ consumo mensal de água pela cultura $\mathrm{i}$, no período $\mathrm{k}$, em mm; $\mathrm{V}_{\mathrm{i}^{-}}$volume de água mensal disponível, $\mathrm{cm}$ mm.ha; $\mathrm{c} \mathrm{R}_{\mathrm{ik}}$ restrição na árca cultivada da cultura $\mathrm{i}$, no período $\mathrm{k}$.

\section{O modelo aplicado}

$\Lambda$ função objetivo para o problema será:

$\max Z=1011 \mathrm{X}_{11}+1011 \mathrm{X}_{12}+\ldots .577 \mathrm{X}_{41}+\ldots .8106 \mathrm{X}_{91}$

Sujeito às seguintes restrições:

(1) disponibilidade mensal de terra

TFRRA.IAN: $\mathrm{X}_{21}+\mathrm{X}_{41}+\mathrm{X}_{91}=7424, \ldots$,

IERRADEL: $\mathrm{X}_{32}+\mathrm{X}_{41}+\mathrm{X}_{61}+\mathrm{X}_{91} \leq 7424$

(2) balanço mensal de área

$\Lambda \mathrm{RE} \Lambda \mathrm{J} \Lambda \mathrm{N}: \mathrm{X}_{21}+\mathrm{X}_{41}+\mathrm{X}_{91}+\mathrm{XM}_{1}=0, \ldots$,

ARFADF7: $\mathrm{X}_{32}+\mathrm{X}_{41}+\mathrm{X}_{61}+\mathrm{X}_{91}-\mathrm{XM}_{12}=0$

(3) balanço de área total cultivada de cada cultura

AREAFPH: $\mathrm{X}_{11}+\mathrm{X}_{12}-\mathrm{XT}_{1}=0, \ldots$,

AREABAN: $\mathrm{X}_{21}-\mathrm{Xl}_{2}=0$

(4) disponibilidade mensal de água

AGUAJAN: $138,6 \mathrm{X}_{21}+238,5 \mathrm{X}_{41}+247,1 \mathrm{X}_{91} \leq 986104, \ldots$,

AGJADE7: $282 \mathrm{X}_{32}+163,1 \mathrm{X}_{41}+217,8 \mathrm{X}_{61}+260,1 \mathrm{X}_{91} \leq 986104$

(5) disponibilidade total de água

AGUATOT: $545 X_{11}+545 X_{12}+887 X_{31}+\ldots+2900 X_{91} \leq 6.664 .445$

(6) restrições devida ao mercado e capacidade de

processamento da indústria local.

$\operatorname{MINX}_{12}: \mathrm{X}_{12} \geq 714 ; \mathrm{MAXX}_{31}: \mathrm{X}_{31} \geq 2854$;

$\operatorname{MAXX}_{32}: X_{32} \geq 2854: \operatorname{MINX}_{12}: X_{12} \geq 714$;

$\operatorname{TOTX}_{3}: \mathrm{X}_{31}+4281 ; \mathrm{MAXX}_{61}: \mathrm{X}_{61} \leq 2141$;

$\operatorname{MINX}_{61}: X_{61} \geq 714 ; \operatorname{MAXX}_{71}: X_{71} \leq 4281$;

$\operatorname{MINX}_{71}: X_{71} \geq 714 ; \operatorname{MAXX}_{81}: X_{81} \leq 357$;

$\operatorname{MINX}_{91}: \mathrm{X}_{91} \geq 714 ; \quad \mathrm{MAXX}_{91}: \mathrm{X}_{91} \leq 2854$.

Tabela 2. Necessidades mensais de irrigação, para cada cultura, em porcentagem da lâmina tolal

\begin{tabular}{|c|c|c|c|c|c|c|c|c|c|}
\hline \multirow{2}{*}{ MESES } & \multicolumn{9}{|c|}{ CUI.TLRA (i) } \\
\hline & 1 & 2 & 3 & 4 & 5 & 6 & 7 & 8 & 9 \\
\hline $\mathrm{J} \Lambda \mathrm{N}$ & & 27,71 & & 30,78 & & & & & 8,52 \\
\hline FEV. & & 38,02 & 25,95 & 27,88 & 25,96 & & & 27,41 & 7,02 \\
\hline MAR. & & 34,27 & 39,98 & 20,30 & 39,98 & & & 39,19 & 7,03 \\
\hline $\mathrm{ABR}$. & & & 34,07 & & 34,07 & & & 33,40 & 6,65 \\
\hline MAIO & 23,29 & & & 16,46 & & & 16,29 & & 6,80 \\
\hline JUN. & 37,57 & & & 24,51 & & & 21.83 & & 6,59 \\
\hline JUL. & 39,14 & & & 31,21 & & & 30,90 & & 7,62 \\
\hline AGO. & & & & 27,82 & & & 30,98 & & 9,34 \\
\hline SET. & 23,40 & & & & & 17,13 & & & 10.06 \\
\hline OUT. & 41,99 & & 28,32 & & & 25,15 & & & 11,06 \\
\hline NOV. & 34,61 & & 39,89 & & & 29,17 & & & 10.13 \\
\hline DF.7. & & & 31,79 & 21,04 & & 28,55 & & & 8,97 \\
\hline
\end{tabular}

A necessidade mensal de irrigação das culturas é apresentada na Tabela 2, como porcentagem de lâmina total necessária durante o ciclo; lais valores foram oblidos pela relação entre a evapotranspiração máxima durante o ciclo e a mensal.

\section{RESULTADOS E DISCUSSÃO}

No projeto Senador Nilo Coelho (PSNC) o maior volume anual de água ofertado aos usuários foi, no ano de 1992, de $66.644 .550 \mathrm{~m}^{3}$ e o maior volume mensal foi de $9.861 .040 \mathrm{~m}^{3}$ (Dantas Neto, 1994). Utilizando-se esses valores como restrições de água e satisfazendo as exigências de áreas máxima e mínima de algumas culturas, o modelo de programação linear proposto resultou no padrão ótimo de cultivo apresentado na Tabela 3, com as respectivos custos marginais.

Tabela 3. Solução ótima para um volume anual de água igual a $66.644 .550 \mathrm{~m}^{3}$

\begin{tabular}{|c|c|c|c|}
\hline CULTURA & CICLO & $\begin{array}{l}\text { ARE.A ÓTIMA } \\
\text { (ha) }\end{array}$ & $\begin{array}{c}\text { CUSTO } \\
\text { MARGINAL }\end{array}$ \\
\hline \multirow[t]{2}{*}{ Feijx̄o Phaseolus } & 1 (maio) & $* * * * * *$ & $-512,40$ \\
\hline & 2 (sctembro) & 714 & -512.40 \\
\hline Feijāo Vignlit & 1 (janeiro) & ******** & $-1200,60$ \\
\hline \multirow[t]{2}{*}{ Melancia } & 1 (fevereiro) & $* * * * * *$ & $-677,30$ \\
\hline & 2 (outubro) & 714 & $-677,30$ \\
\hline \multirow[t]{2}{*}{ Milho } & 1 (maio) & *⿻:k:k: & $-1589,30$ \\
\hline & 2 (dezembro) & 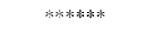 & -1589.30 \\
\hline Melăo & 1 (fevereiro) & $* * * * * * *$ & $-1497,50$ \\
\hline Pimentäo & 1 (sctembro) & 714 & $-1309,70$ \\
\hline Tomatc & 1 (maio) & 4281 & 1012,30 \\
\hline Cebola & 1 (fevereiro) & 357 & 4079,80 \\
\hline Banana & 1 (ano total) & 818 & $* * * * * *$ \\
\hline \multicolumn{2}{|c|}{ Total } & 7598 & \\
\hline \multicolumn{2}{|c|}{ Rendn líquida (US\$) } & 22.634 .1044 & \\
\hline
\end{tabular}

A cultura do feijão Phaseolus, com plantio em maio, não foi recomendada. Nesta época, só será recomendado-se a disponibilidade anual de água for superior a $95.000 .000 \mathrm{~m}^{3}$; contudo, devido à restrição de área mínima para satisfazer a demanda interna, foi indicada uma área de 714 ha desta cultura, com plantio em setembro. O valor negativo do custo marginal apresentado na Tabela 3 está associado à cultura não recomendada para cultivo e se refere à redução da renda líquida por unidade de área cultivada; por exemplo, para cada hectare de feijão Phaseolus cultivado em maio haverá redução de US\$512,40 na renda líquida, se o volume anual de água disponível for igual a $66.644 .550 \mathrm{~m}^{3}$. A melancia plantada em outubro tem restrições de área mínima (714 ha) e máxima (4281 ha), devido à disponibilidade de mercado. O modelo previu apenas o plantio de 714 ha para satisfazer o mínimo requerido. A área de melancia plantada em outubro só deverá ser superior a 714ha para volumes de água superiores $66.644 .550 \mathrm{~m}^{3}$. As culturas tomate e cebola apresentam custos marginais positivos indicando que, caso ocorra aumento em suas áreas máximas de plantio haverá, consequentemente, maior rendimento financeiro.

A Tabela 4 apresenta os volumes anuais de água utilizado pelas culturas em função dos volumes disponíveis e seus respectivos preços-sombra.

Uma análise da Tabela 4 mostra que, para volumes de água disponíveis inferiores a $96.874 .460 \mathrm{~m}^{3}$, ocorreu utilização plena deste recurso na solução-ólima decorrendo, para cada 
Tabela 4. Volumes anuais de água utilizados em função dos volumes disponíveis e respectivos preços-sombra

\begin{tabular}{|c|c|c|c|c|}
\hline \multicolumn{4}{|c|}{ VOLUME DE $\Lambda$ GU $A$ ANU $\Lambda \mathrm{L}\left(\mathrm{m}^{3}\right)$} & \multirow{2}{*}{$\begin{array}{c}\text { PREÇO-SOMBRA } \\
\left(\text { US } \$ \mathrm{~m}^{-3}\right)\end{array}$} \\
\hline Disponível & Utilicado & Minimo & Máximo & \\
\hline 40.440 .000 & 40.440 .000 & 40.433 .820 & 43.375 .500 & 0.775 \\
\hline 58.000000 & 58.000 .000 & 43.375 .500 & 63.636 .060 & 0.458 \\
\hline 66.644 .550 & 66.644 .550 & 63.636 .060 & 67.918 .200 & 0.280 \\
\hline 80.000 .000 & 80.000 .000 & 72.599 .840 & 89.987 .160 & 0.203 \\
\hline 95.000 .000 & 95.000 .000 & 94.957 .670 & 96.874 .460 & 0.186 \\
\hline 96.874 .460 & 96.874 .460 & 96.874 .460 & ******; & 0,000 \\
\hline
\end{tabular}

volume, um valor de preço-sombra (custo de oportunidade de utilizar certo volume de água). Volumes superiores a este apresentam folga e, como o excesso do recurso, volume de água anual, não é utilizado para aumentar a renda, o preço-sombra é zero.

O preço-sombra é válido dentro de certos limites de disponibilidade do recurso, os quais são indicados na Tabela 4 como volumes anuais de água mínimo e máximo. Para o exemplo, os preços-sombra da água são inferiores a US\$ $0,775 . \mathrm{m}^{3}$ O volume anual de água disponível deixa de ser uma restrição efetiva à produção quando seu valor é no mínimo de $96.874 .460 \mathrm{~m}^{3}$.

Em perímetros irrigados o preço-sombra da água representa o valor que o irrigante poderá estar disposto a pagar para ter disponível, na sua parcela, uma unidade de volume adicional porque, economicamente, o irrigante deverá pagar valor no máximo igual à renda líquida gerada por uma unidade adicional do recurso. Por exemplo, para volumes disponíveis entre 72.599 .840 e $89.987 .160 \mathrm{~m}^{3}$, o irrigante poderá pagar até US $\$ 0,203$ para adquirir mais $1 \mathrm{~m}^{3}$ de água. Para cada unidade de volume a menos, ocorrerá redução de US $\$ 0,203$ na renda líquida. Dentro dos limites máximo e mínimo apresentados para os volumes disponíveis (Tabela 4) não mudam as variáveis básicas (culturas) encontradas na solução-ótima; entretanto, mudam seus valores (áreas cultivadas).

A análise de sensibilidade da função-objetivo (Tabela 5) mostra como se comportaria a solução-ótima caso houvesse variação na receita marginal de cada cultura. As informações são válidas para um volume de água anual disponível de $66.644 .550 \mathrm{~m}^{3}$. O feijão Phaseolus e a melancia proporcionaram receita marginal inferior a US $\$ 1523,34 \mathrm{ha}^{-1}$ e US $\$ 3479,4$ ha $^{-1}$ respectivamente; no entanto, devido às restrições de mercado, o modelo recomenda o plantio de 714 ha de feijão em setembro e de 714 ha de melancia em outubro. A cebola pode proporcionar até um mínimo US\$ 2303,20 ha ${ }^{1}$ e sua área ólima de plantio será de 357 ha (valor máximo permitido); a área de banana, de 818ha continuará sendo ótima enquanto a sua receita marginal permanece entre US\$ $\$ 891,50$ e US\$13274,70.ha-1 . A mesma interpretação se dá às demais culturas.

Tabela 5. Resultados da análise de sensibilidade da função-objetivo

\begin{tabular}{|c|c|c|c|c|}
\hline \multirow[b]{2}{*}{ CUITURAS } & \multirow{2}{*}{$\begin{array}{l}\text { F́POCA DE } \\
\text { PI.ANTIO }\end{array}$} & \multicolumn{3}{|c|}{ VAIOR DA RFCFITA MARGINAI (US\$.ha') } \\
\hline & & F. OB.JETIVO & MÍNIMO & MÁXTMO \\
\hline F. Phascolus & setembro & 1011,00 & ******* & 1523,40 \\
\hline Mclancia & outubro & 1802,00 & $* * * * * *$ & 2479,40 \\
\hline Pimentảo & setembro & 823,00 & ****** & 2132,70 \\
\hline Tomate & maio & 2600,00 & 1587,70 & $* * * * *$ \\
\hline Ccbola & fevereiro & 6383,00 & 2303,20 & ****** \\
\hline Вапаnа & ano todo & 8106,00 & 5891,50 & 13274,70 \\
\hline
\end{tabular}

\section{CONCLUSÕES}

A maximização dos lucros da área de colonização do projeto Nilo Coelho, obtida com o modelo de programação linear, foi de US\$22.634.044, utilizando-se o seguinte padrão de cullivo: feijão Phaseolus (714 ha), melancia (714 ha), pimentão (714 ha), tomate (4281 ha), cebola (357 ha) e banana (818 ha). A disponibilidade anual de água foi restrição atuante e apresentou preço-sombra de 0,280 (US $\$ . \mathrm{m}^{-3}$ ). O volume anual de água disponível deixa de ser uma restrição efetiva à produção quando seu valor for de no mínimo $96.874 .460 \mathrm{~m}^{3}$

\section{REFERÊNCIAS BIBLIOGRÁFICAS}

ALBUQUERQUE FILHO, J.L. Pesquisa operacional aplicada à agricultura irrigada. IN: CURSO DE ESPECIALIZAÇÃO EM IRRIGAÇÄO, 1986. Apostila. Campina Grande, PROINE, UFPb, 1986. $27 \mathrm{p}$.

BALTRA, C.O. A utilização da informática na irrigação. ITEM, Brasília, n.28, p.13-15,1982.

DANTAS NETO, J. Modelos de decisão para otimização do padrão de cultivo em áreas irrigadas, baseados nas funções de resposta das culturas à água. Botucatu: UNESP, 1994. 125 p. Tese (Doulorado)-Faculdade de Ciências Agronômicas, Universidade Estadual Paulista, 1994.

EVIA, C.V.J. Programacion lineal para la formulación de planos de cultivos en los distritos de riego del pais. Rec. Hidraul., Mexico, v.4, p.562-577, 1975.

FRIZZONE, J.A. Programação matemática aplicada a projetos hidroagrícolas. VIÇ.OSA: SBEA. 1995. 29p. (Sociedade Brasileira de Engenharia Agrícola, Mini- Curso, 95-03).

WINDSOR, J.S.; CHOW, V.T. Model for farm irrigation in humid areas. J. irrig. Drain. Eng.,New York, v. 97, p.291303, 1971. 